\title{
Chinese Tibetan Guozhuang Dance from the Perspective of Sports
}

\author{
Qian Liu ${ }^{1}$, Jianqiang Wang ${ }^{1} \&$ Yinan Qin ${ }^{1}$ \\ ${ }^{1}$ Department of Sports, College of Arts and Sports, Sichuan Agricultural University, Ya'an, China \\ Correspondence: Qian Liu, Department of Sports, College of Arts and Sports, Sichuan Agricultural University, \\ Ya'an 625014, Sichuan, China. E-mail: lydia2000@yeah.net
}

Received: February 15, $2012 \quad$ Accepted: March 19, $2012 \quad$ Published: June 1, 2012

doi:10.5539/ass.v8n7p240

URL: http://dx.doi.org/10.5539/ass.v8n7p240

\begin{abstract}
The authors study Chinese Tibetan Guozhuang dance from the perspective of sports. And they discover that Guozhuang dance has such features as national tradition, holistic arts and public entertainment, etc., and is a kind of national traditional sports activity that has low requirements for sports field, has robust and straight movement, no much exercise intensity and in which the movement amount can be randomly adjusted to satisfy the movement aesthetic requirement. In the process of development, it has manifested such important historical and cultural value as strengthening Chinese Tibetan national cohesion, extending and inheriting Chinese Tibetan culture and promoting different national cultural communication, etc. In the realistic life of the areas where Chinese Tibetans inhabit, Guozhuang dance also has such important sports value as promoting Chinese Tibetan people's physical and psychological health, facilitating social sports, school sports and sports tourism development, etc.

Keywords: national traditional sports, perspective of sports, Chinese Tibetan, Guozhuang dance

Traditional Chinese Tibetan sports have the same source and different flows with its national dance and the phenomenon of integration of traditional sports and arts exists generally. The artistic pattern that reflects life and expresses ideological feelings by means of organizing processing and typical human body movement and modeling, namely, also falls within the scope of traditional Tibetan national sports. This sort of social activity passes on from one generation to another that regards body exercise as the basic means and regards strengthening human body quality and enriching social and cultural life as the purpose. Chinese Tibetan Guozhuang dance is a sort of movement item that integrates dance, sports and music into one whole. That is to say, Guozhuang dance is not only a sort of dance, but also a sort of sports, which has the effects of sports in the form of dance, so it is more appropriate to term it as a sort of dance sports. On May 20, 2006, Guozhuang dance was approved by the State of Council and listed into the first batch of the national non-material cultural heritage directory (The State Council, 2006). However, due to a variety of reasons, so far, a relatively in-depth study has still been lacking on this sort of national traditional sports activity. This paper is aimed to enhance people's knowledge in Chinese Tibetan Guozhuang dance from the perspective of sports.
\end{abstract}

\section{Features of Chinese Tibetan Guozhuang Dance}

\subsection{National Tradition}

National traditional sports are the traditional culture that has been created and accumulated in the long history by our ancestor, fully embody the common cultural value concept and aesthetic ideal of all nations in China, a traditional cultural phenomenon that is closely related with the social characteristics, economic life, religious rite, customs and habits and historical culture of all nations in China, and reflect the vigor of life and creativity of the Chinese nation (Ni Yike \& Hu Xiaoming, 2006) Guozhuang is a stone or a triangle that supports the iron pan and copper pot in the fire pond and people always move around the fire pond, so this sort of dance is called Guozhuang dance. Guozhuang dance per se has the property of primitive religious rite and its content is manifested as collection of the original totem culture, such as the culture of oath of alliance. It stretches across the three large fields of sports, arts and education, integrates body exercise, dance and music into one whole and retains deep Chinese Tibetan features and local flavor. Guozhuang dance has the feeling of holistic movement and when it is performed, there is not any musical instrument accompanied. In the vigorous and firm folk song sound, people move around a circle and dance together and take a bighearted, firm and steady Guozhuang step, which indicate simple and honest and a heavy personal characteristics and simple and powerful, rough and bold 
national traditional characteristics with hardness and softness.

\subsection{Holistic Artistic Feature}

The artistic feature of Guozhuang dance is mainly reflected in music, movement and alteration of formation as well as orderliness, unification and harmony of the dancing group. The number of the people who participate in the dance is changed from several to dozens of people and even as much as hundreds and thousands of people. The dancers dance hand in hand with each other. Either men and women respectively form an arc or a circle and transit from a circle with a single form to the double-layer concentric circle, multi-layer concentric circle, side-by-side circle, crossing circle and separation circle, or men and women can mix together to form the above formations. One pattern includes one song and one dance from slowness to fastness or firstly from slowness to fastness and then to an end in adagio. After a dance and a song come to an end, all the dancers shout out together with the sound of "Ya", suddenly accelerate their speed in accompany with the lucid and lively rhythm of the tune, and, in the meantime, spread out their two arms, lean to one side, twist their waist and stride to jump up. The other pattern is to walk in adagio and then immediately turn to a dance without outburst of rhythm after several parts of dance. The rhythm of the allegro part is urgent and the emotion of dancers is quite fierce, in which the shouting voice of "e hei hei" bursts out from the group. Both of the two patterns are firstly singing and dancing in the adagio, and, in the meantime, take a footwork of "jumping on the two feet and stepping backward with one foot" and winding forward. When in the allegro, dancers shouting "Qu, qu qu" at the time of making highly difficult skills of "leaping forward by lying on the ground", and move forward in a fast step, which highlights the beauty of "one side movement" with large ups and downs.

\subsection{Public Entertainment}

Guozhuang dance means that several people come together and finish a variety of movements with the same rhythm. In the process of the activity, the coordinative cooperation of all participants, the physical and psychological enjoyment generated in the process of the activity and the emotional expression realized in the process are all the purposes of this activity. Hence, one of the cultural attributes of Guozhuang dance is its typical non utilitarianism, or at least little utilitarianism sports activities. in this sort of activity, since there does not exist any struggle of victory or defeat among the participants, there is no physical extreme tiredness and high pressure of emotion that are necessarily caused in the competitive sports. A common person is able to participate in the dance since it has no particular requirement for the number of participants, the sports occasion or the facility conditions, suits with different ages and levels of people, a kind of cultural entertainment activity that is greatly favored by the Chinese Tibetan people and that has high public participation and that has the obvious characteristics of being delighted to see and hear by the vast majority of people. The interactive cooperation form and the lucid and lively music and libretto make the dance endowed with interestingness and entertainment ingredients and enable people to make physical and psychological adjustment and emotion release at the spare time of the strained learning and working.

\section{The Kinematics Features of Chinese Tibetan Guozhuang Dance}

The movement of Guozhuang dance is mainly derived from the life and production, has strong labor sense, has the major form of flexion and extension, is lucid and lively, powerful and heavy and is accelerated gradually from slowness. The movement is driven with emotion, with integration of motion and emotion and builds up the particular amorous feelings and connotation of Guozhuang dance. Its performance is enthusiastic and unrestrained, joyous and vigorously and has continuity and pause. The upper body movement mainly includes crossing the hands across the chest, relying on the back, bending the arms and swinging the hands upwards. The trunk and the upper limb move with the steps and the sleeves are waved up and down, surging and vigorous. The foot step mainly includes the action of lifting, jumping, stamping, striding, stepping and rubbing, etc. Guozhuang dance is of great momentum, with strong skills, has no strict demand on the texture of the sports occasion and it is OK so long as the ground is smooth and even. In performance of Guozhuang dance, men and women movement around a circle and sing and dance from the right to the left. The movement range of men is relatively large and they are just like a tercel hovering and flying when they extend their arms. The movement range of women is relatively small and they whirl just as a phoenix winging and flying, which has the features of bodybuilding, lucid and lively of sports movement. The biggest difference of Guozhuang dance from other folk dance forms is that, the limbs are moved along the same side, the left hand the left foot move simultaneously and the right hand the right foot move simultaneously. Through observation, we find that, in the study on the skill features of Guozhuang dance, we should not only pay high attention to the relatively small movements that have been formed in the evolvement of the long history of Guozhuang dance, but also focus on the particular movement styles that are presented by these movements. In the meanwhile, we should also note that the above 
skill features of Guozhuang dance have enabled the movement of Guozhuang dance to have great variability and re-creation space and to create again more various new movements under direction of the skill principles that have been formed for the last few years so as to enrich its movement content. This is exactly the important security for this sort of activity to be continuously development, adapt to the modernization transition and better serve the realistic life.

The movement strength of Chinese Tibetan Guozhuang dance is not large and is a sort of body movement led by aerobic metabolism. The movement time that determines the amount of exercise can be either long or short and the participants can participate in the movement from beginning to the end together with the team and can also participate midway or withdraw according to their own physical condition. This movement strength and movement amount is suitable and can be adjusted by means of controlling the speed and range of movement and the time of exercise, with combination of enjoyment and national dance, which not only enables this movement to have high security and good bodybuilding effect, but also enables this movement to become a bodybuilding project which men and women and the old and the young can all participate in and are willing to engage, so that it has the extensive adaptability characteristics.

Today, a large number of modern sports items have higher demand on aesthetics, such as, artistic gymnastics, aerobics, synchronized swimming, figure skating and martial art, etc. Yet, Guozhuang dance is on the top of beauty, and its movement process is also the process of creating beauty and it expresses and communicates with a body language, presenting a sort of aesthetic feeling that can't be expressed. Participation in Guozhuang dance can make the body appearance of human better balanced and more harmonious, the human body more energetic and delicate and the movement alternate between kindness and severity. This can not only satisfy the will of an individual to realize self beauty, but can also become the aesthetic object of others. In addition, the position matching of the group of Guozhuang dance and change of the formation of the team, accompanied with a corresponding song, form a strong, delicate and rich dynamic picture. At the time of letting others appreciating Guozhuang dance, the dance also combines the formal beauty, posture beauty, movement beauty and clothing beauty that people see and the song beauty people hear, make adjustment in terms of emotion, let others appreciate and appreciate oneself to enjoy beauty.

\section{Major Historical and Cultural Value of "Dancing Guozhuang" by Chinese Tibetan People}

\subsection{To Strengthen National Cohesion}

Chinese Tibet is a nomadic people which is distributed mainly in the plateau area. During the period of lower productivity in which self-sufficient livestock economy is the major economy, the particular background of social production and life determines that individuals within the nation have no high dependence upon each other and have no close connection with each. Thus, in the historical development of the particular period of Chinese Tibet, the common activities that enable the individuals of the nation gather together all have the important effect of maintaining national identity and protecting national solidarity and unification. Especially, Tibetan people are scattered everywhere in the ordinary period, but "Guozhuang dance" unites them together. They have the same movement with an impulse, so "Guozhuang dance" helps to adjust social order and join scattered agricultural ald livestock life (Zhou Jin, 2002). During this historical period, Chinese Tibetan people as individuals take part in such activities as "Guozhuang dance" to expand their social exchange and promote improvement of their personal socialization, which is helpful for progress and development of individuals and the whole nation. "Guozhuang dance" is a sort of equal, coordinated and harmonious exchange and is a kind of experience and feeling of the national culture. When people participate in "Guozhuang dance", a sort of closeness and identity feeling might emerge and the consciousness of deriving from the same root underlying in the dance naturally collects the individuals. The same ancestor, the same place of origin, the same religious belief, the same pray, the same will and the sense of belonging and dependence of the same root and the same ancestor make Chinese Tibetan "Guozhuang dance" filled with a sort of joyous and peaceful national solidarity flavor and make individuals filled with confidence and power in the nation and the society. In this environment, the spiritual adaptability and sense of satisfaction that are brought by a variety of factors to the individuals objectively reflect the emotional condition of individuals so as to satisfy the need of interpersonal exchange and cooperation, further effectively promote social exchange attitude and capacity of individuals and development of friendly and civilized behaviors in the process of exchange, strengthen sense of identity of the national groups by means of dredging emotion and mutually understanding and conveying information and further obtain corresponding national cohesion (Hu Zhuosheng, 2008).

\subsection{Extension and Heritage of Particular Chinese Tibetan Culture}

National peculiarity is the fundamental feature of non-material cultural heritage and is the "special value" that is 
deeply rooted in cultural tradition or relevant regional culture (Xu Haiyan, 2006). Chinese Tibetan "Guozhuang dance" is absolutely not merely a pure body activity of bouncing and vivacious, but is a sort of body movement form that is highly related with spirit, belief, morality, aesthetic concept and value orientation height, based on knowledge, theory, method and skill, and has extremely abundant cultural connotation. Other nations also have somebody activities that are similar to Chinese Tibetan "Guozhuang dance", such as, dancing by moving across a campfire by Ma sa people in Liangshan, Sichuan Province and "beating and dancing" by the minority of Yunnan Province. All these traditional sports activities have extremely abundant cultural connotation, reflect the spiritual belief, moral values and aesthetic interests of the nations, and, meanwhile, are closely connected with such cultural features as their language, national characteristics, national personality and life style, etc., so as to really reflect the cultural personality of all nations in continuous development and progress. There is no exception to Chinese Tibetan "Guozhuang dance", but it has an obvious national difference from other seemingly similar body movement forms, which is essentially the cultural difference. Culture is a sort of life style (Ouyang Zhesheng, 1998) that is formed by civilization and different groups have different culture. Once culture is formed, a strong internal spiritual power will be formed, which can not only maintain the internal order of the group, but can also provide ideological concept, moral standard and value norm for members in the group, so as to direct and standardize their behaviors (Chen Xingliang, Liu Rui \& et al., 2007). Culture is also the blood vessel and soul of a nation, since the particular culture and tradition of different nations is the condition for its survival and extension. Chinese Tibetan "Guozhuang dance" is a comprehensive national cultural activity that regards body activity as a carrier and has multiple cultural connotations and cultural appeals. In the long historical development process of Chinese Tibet, "Guozhuang dance" that has abundant cultural connotation is passed down from one generation to another, which is not only extension of body movement form (actually, extension of this body movement form is merely regarded as a carrier), but more importantly, this dance holds together and inherits the particular Chinese Tibetan culture in this extension.

\subsection{Promoting Cultural Communication and Harmonious Development of Different Nations}

Nation is distinguished by culture and different nations have different culture. Assuming that different nations fail to find out similarity in terms of culture, mutually respect the culture of each other or learn and borrow mutual cultural achievements, then it is difficult to break through the national boundary. On the contrary, if different nations have cultural communication and identity, then the national difference might be played down by the sense of identity and sense of harmony and different nations might get closer to each other. It is exactly by means of cultural communication and promoting cultural integration and combination that the Chinese nation has the pattern of "integrated diversity and unity in diversity" is formed in the progress of history. That is to say, different nationalities not only have similarity of Chinese culture, but also have their own cultural personality and the interactive relationship of "I'm in you and you're in me" is formed among different nationalities. Thus, the 56 nationalities with different sources of origin come together on the Chinese land, and the fundamental reason is that in the long history, cultural communication and integration of different nationalities have gradually generated the great Chinese cultural system constituted by a lot of national culture. This history does not only tell us that the most fundamental factor to maintain Chinese solidarity and unification is the great Chinese culture with great tolerance, but also tells us that cultural communication within all nationalities of the Chinese nation is the most fundamental cause for promoting gradual formation of the Chinese nation and Chinese culture. The history also tells us that, in different national cultural communication, those cultural patterns that are more likely to bring happiness and satisfy people's aesthetic demand and meanwhile, are less likely to arouse contradiction and conflict in terms of spirit and utility, are more likely to rapidly disseminate in different nations and gradually integrate into the cultural life of other nations. As a sort of cultural activity that has clear-cut entertainment feature, "Guozhuang dance" has the above feature. Therefore, in the long history of development of Chinese Tibetan "Guozhuang dance", the Tibetan people not only resort to this activity that has clear-cut national cultural features to prove existence of Chinese Tibet to other neighboring nations, but also regard this dance as a bridge to promote cultural communication of Chinese Tibetan people with other nations to make a closer national relationship.

\section{The Major Sports Value of Chinese Tibetan Guozhuang Dance}

\subsection{Promoting the Physical and Psychological Health of Chinese Tibetan People}

Chinese Tibetan Guozhuang dance has strong characteristics of self-entertainment and amusement of others. The so-called self-entertainment means that the participants realize expressing their emotion and entertaining their body and mind by means of body movement, while amusement of others means that satisfaction given to the external audience can arouse the same feeling of the audience. This sort of activity has not only enhanced the socialization degree of individuals and promoted formation of national identity, but has also enabled people to 
fully experience and share the humanistic joy of mutual coordination, mutual collaboration, mutual care and friendly exchange between people and people and between people and the group. Then, choice of the value target of Guozhuang dance is obviously a sort of non-productive game entertainment activity and the purpose of this activity itself is to enable people to obtain physical and psychological job and happiness. Therefore, under direction of this purpose and in the long development process of Guozhuang dance, it gradually becomes possible to enhance the entertainment style of the enjoyment of Guozhuang dance by means of rhythmic music and all sorts of rhythmic coordination movements, the cooperation position of the group and the alteration of the team pattern, etc. and a perfect effect of cheering the body and the mind is received. In the process of the entire activity, clear-cut rhythm feeling, the coordination feeling of the body, the perfect body feeling gradually caused by the activity and the tacit understanding and collaboration of participants enable this activity to have high interestingness and enjoyment. Through the Guozhuang dance, the emotion of human being gets expressed, their negative emotion is released, their psychology is satisfied and their spirit is resonated, which, altogether, makes people obtain confidence and strengthens the national cohesion and centripetal force.

Guozhuang dance is robust in movement, tall and straight, which not only presents the dance posture, but also focuses on expression of emotion. The dance posture of Guozhuang dance is smooth and natural, beautiful and elegant, not only embodying the simple and kind, industrious and brave, enthusiastic and bold national characteristics, but also having certain vigor and the changing movement of running and jumping, with a large range of movement and obvious sports dance training value and exercise value. Guozhuang dance reflects the style of Chinese Tibetan folk sports both in terms of the dance posture changes of performers and in terms of the movement rhythm of performers, and its effect of bodybuilding goes without saying. In Chinese Tibetan area where both material life and spiritual life of people are relatively deficient, the prominent self-entertaining and self-enjoyment function and bodybuilding function of Guozhuang dance obviously effectively enriches the cultural life of Chinese Tibetan people, which is helpful to promote the physical and psychological health of Chinese Tibetan people and becomes the important content of Chinese Tibetan cultural development.

\subsection{Facilitating Development of Social Sports}

In June 1995, the State Council promulgated "outline of the nationwide bodybuilding plan", with the purpose of trying to build a national bodybuilding system with Chinese characteristics as soon as possible, further promote wide development of Chinese social sports so as to enhance Chinese people's body health in a comprehensive way and lay a good human resource foundation for further promoting the modernization progress of China. However, as a result of such factors as historical and economic development, the development level of Chinese social and sports is relatively low and sports occasion and sports facilities are relatively in deficiency. Insufficient sports occasion is the reason that is only second to "lack of time" which affects Chinese urban and rural citizens in participation in sports exercise (Zhang, Faqiang, 1999). According to the fifth national sports occasion survey bulletin that was promulgated by the General Administration of Sport of China in 2005, an average of 10000 people owned 8.3 sports stadiums in Beijing and the per capita sports area was $1.71 \mathrm{~m}^{2}$ in Shanghai. Even the cities that have relatively high economic and social development level have such a situation, let alone the insufficient condition in the vast area of Chinese rural areas. Nonetheless, the strong requirement to enhance the living quality and physical and psychological health level of citizens does not allow us to wait to largely facilitate social sports development until the national economy is greatly developed and the national financial power and residents' consumption level can better satisfy the requirement for sports occasion and facilities. Then, the most realistic and also most effective solution is to seek for sports means that have low requirement for sports occasion and facilities, have strong bodybuilding and entertainment characteristics, are easy to promote and are able to better promote the physical and psychological as well as spiritual health of participants. Promotion of these sports means in a larger scope helps to facilitate urban and rural social sports development. Just as has been mentioned above, Guozhuang dance that is popular in Tibetan area has this obvious feature. Therefore, it is totally possible for us to further enrich the movement content and strengthen its bodybuilding level so as to promote its development within a larger scope at the time when we effectively explore and sort out Guozhuang dance. This can not only help to promote effective development of social sports in pastoral areas where the vast majority of Chinese Tibetans gather together, but can also gradually make Tibetan people walk into life of other nationalities. It is possible to facilitate development of rural social sports in a more extensive area which can also be promoted in urban areas to make Guozhuang dance become a convenient and effective sports and life style for urban residents and make the ancient Guozhuang dance in Chinese Tibet fully exhibit its times value in promoting development of the current social sports in China.

\subsection{Realizing Interactive Development with School Education}

School education is the major component of the national bodybuilding and plays a quite crucial role in the 
national bodybuilding. In the rural areas in China, especially in economically underdeveloped western rural areas, the sports occasion, facilities and teachers of school sports are in great deficiency. Yet, according to the school sports reform that was initiated at the beginning of the $21^{\text {st }}$ Century, the schools can develop sports course resources in light of their reality and have greater autonomous rights in terms of textbook selection so as to try not to or seldom to be restrained by the sports occasion, facilities and teacher conditions on the basis of the current economic development level and to effectively develop school sports. National traditional sports is an important component of the national culture in China, so bringing the national traditional sports into school sports is of profound historical significance and realistic significance to carry forward and inherit the excellent national traditional sports culture. Therefore, western rural schools can bring some national traditional sports items that comply with the reality into the sports course resources after the textbook processing of the national traditional sports in accordance with local economic development level and natural and humanistic characteristics, etc. Guozhuang dance that is popular in Chinese Tibetan area is exactly this sort of national traditional sports activities that is extensively employed in school sports, especially in rural schools in Tibetan areas and the surrounding areas, so as to promote effective development of school sports. In the meantime, school sports are the foundation of lifelong sports and the movement interest and habits are the precondition to facilitate autonomous learning and lifelong persistent exercise of students. In teaching of Guozhuang dance, we can create a loose, harmonious, peaceful and vigorous atmosphere to encourage students to enthusiastically and courageously manifest themselves and present themselves, stimulate students' interest in learning, enable them to form the habit of conscious exercise and form a lifelong sports consciousness (Zhang Bingsong \& Zhao Wentao, 2007).

Just as basketball and volleyball that were originated in European and American countries and such movement items as martial arts in China, Guozhuang dance will gradually establish and enrich its own theory and method during its undergoing the process of going to the schools and becoming the school sports resources and will be more widely spread in the whole society by the means of school sports. Thus, walking into school sports is an extremely important approach and method for a large majority of sports items to acquire effective development. When we give the textbook processing to Chinese Tibetan Guozhuang dance and bring it into the school sports, it is necessary that we will not only facilitate effective development of school sports, but also promote development of Guozhuang dance and realize interactive development of school sports and Guozhuang dance.

\subsection{Forming a Benign Circulation with Sports Tourism Development}

In the contemporary world, combination of sports and tourism is a trend and sports tourism has become a newly emerging industry. From the perspective of modern economics, national traditional sports, as a sort of culture, if national traditional sports is merely regarded as an object of academic study, then it is difficult to tap its real value. Only if it is regarded as a sort of resource to participate in social and economic culture, can it really embody its real value in allocation of resources (Liu Shaoying \& Zhao Zhiqiang, 2008). In tourism activities, there is such a rule that deserves our high attention, that is, the greater difference the natural scenery and human landscape has from the natural and human environment where the tourists live for long and which they are familiar with, the more likely it is to stimulate the tourism interests of tourists, effectively arouse tourism impulse and get the tourists satisfied. It is exactly this rule that makes different natural scenery and human landscape have different degrees of tourism value as for different tourists and makes the minority traditional sports activities that have different characteristics and different national features become the important factor to facilitate sports tourism development (Wang Tianjun, 2003). The regionalism, national feature and interactive participation of Chinese Tibetan Guozhuang dance are the advantages to promote sports tourism development. Whether people watch the dance by themselves or participate in the dance personally, they can all sincerely appreciate the truth of Chinese Tibetan culture on the plateau, enjoy the national sentiment of Chinese Tibet, acquire physical and psychological joy and satisfy the psychology of seeking for newness, happiness, knowledge, movement and curiousness of tourists (Jiang Ming \& Zhou Xinlin, 2005). Combination of cultural tourism and bodybuilding entertainment in an organic way not only enriches tourism activity content, but also leaves more profound impression upon tourists and makes the tourists have a feeling of enjoying themselves so much as to forget to leave in an aftertaste. Thus, full exploration and development of the sports and tourism value of Guozhuang dance is of great significance to gradually form Tibetan sports tourism. In the meantime, Guozhuang dance has the important characteristics of low requirement on sports occasion, being easy to learn and especially sustainable development, which altogether make it become an important sports and tourism resource with great value and efficiency. This sports and tourism resource value of Guozhuang dance as a sort of Chinese Tibetan traditional sports activity will be fully explored. Then, development of sports and tourism will effectively promote local economic and social development and Guozhuang dance will, thereby, receive high attention and 
gain better development conditions owing to the full realization of this sort of value, which will then form a benign circulation of sports and tourism development.

\section{Concluding Remark}

In one word, Chinese Tibetan Guozhuang dance has the features of national tradition, holistic arts and public entertainment, and is a kind of national traditional sports activity that has low requirements for sports field, has robust and straight movement, no much exercise intensity and in which the movement amount can be randomly adjusted to satisfy the movement aesthetic requirement. It is not only the excellent representative of Chinese Tibetan brilliant dance culture with a long history, but is also a project that has the longest history in existing national sports culture, is most likely to reflect Chinese Tibetan sports features and has the most profound influences. Thus, it is an extremely precious artistic wealth in the whole human society and plays an important role in the social life of Chinese Tibetan people. As a sort of national traditional sports movement, it is without doubt that Guozhuang dance has effectively promoted the social intercourse of people, enriched people's cultural life and promoted the physical and psychological health of people as for Chinese Tibetan individuals of one generation after another. Today when modernization is developed continuously, due to the wide adaptability and effectiveness of this particular sort of national traditional sports movement, it can not only become an effective sports means to facilitate Chinese social sports' further development, but can also be brought into school education as a sort of curriculum source so as to promote effective development of school sports. At the same time, its sports tourism value can be fully explored to promote development of Tibetan sports tourism. Thus, a study on Chinese Tibetan Guozhuang sports from the perspective of sports is of great realistic significance to inherit and carry forward this national sports treasure.

\section{References}

Chen, Xingliang, Liu, Rui et al. (2007). Heritage of Traditional Qiang Culture as Seen from Qiang People's Traditional Sports. Journal of Wuhan Institute of Physical Education, 41(10), 90-93.

$\mathrm{Hu}$, Zhuosheng. (2008). Bringing Guozhuang Dance into Study of National Higher School Sports. Health Vocational Education, 26(3), 30-33.

Jiang, Ming, \& Zhou, Xinlin. (2005). On Tibetan "Guo Zhuang" Health Value. Journal of Kangding Nationality Teachers College, (5), 20-21.

Liu, Shaoying, \& Zhao, Zhiqiang. (2008). Discussion on the Problem and Countermeasure of Exploring the Nation Tradition sports tour in Wuling Mountain. Journal of Xi'an Physical Education University, 25(1), $46-48$.

Ni, Yike, \& Hu, Xiaoming. (2006). Discussion on Protection of National Traditional Sport Culture Heritage. China Sport Science, 26(8), 66.

Ouyang, Zhesheng. (1998). Our Attitude towards Western Modern Civilization, Collected Works of Hu Shi. Beijing: Peking University Press, 1-6.

Wang, Tianjun. (2003). Comparative Advantages and Development Strategies of Sports Tourism in Minority Area in Xinjiang. Beijing: Nationalities Publishing House, 141-148.

Xu, Haiyan. (2006). Discussion on Nonphysical Cultural Heritage Protection. Culture Journal, (2), 71-75.

Zhang, Bingsong, \& Zhao, Wentao. (2007). Brief Analysis of Application of "Guozhuang" in Sports and Health Class. China Education Innovation Herald, (12), 73.

Zhang, Faqiang. (1999). Findings Report on a Present Survey of Social Sport in China. Sport Science, (1), 4-7.

Zhou, Jin. (2002). The Social Function of Guo Zhuang Dance in Tibetan Region. Department of Sociology, Sichuan University, 31-37. 